Check for updates

Cite this: RSC Adv., 2019, 9, 28061

\title{
Chondroitin: a natural biomarker with immense biomedical applications
}

\begin{abstract}
Dilipkumar Pal (iD *a and Supriyo Saha (iD b
Naturally extracted glycosaminoglycan chondroitin sulphate is the reactive product of $\mathrm{N}$ acetylgalactosamine and D-glucuronic acid. Chondroitin sulfate (CS) extracted from Scophthalmus maximus, $H$. scabra, E. fraudatrix, M. magnum, and $H$. mexicana has shown remarkable anticoagulant, articular cartilage repair, corneal lesion healing, antidiabetic, and antiproliferative effects. Also, platinum and strontium nanoparticles of chondroitin sulfate are effective in osteoarthritis and exert anti-HSV2 and anti-angiogenic properties. A combination of chondroitin sulfate and RNA lipolexes demonstrates gene silencing effects in liver fibrosis. Chondroitin sulfate has also been used as a carrier for loxoprofen hydrogel preparation. Oligosaccharides of chondroitin sulfate showed effective inhibition of bovine testicular hyaluronidase enzyme as an antibacterial agent during pregnancy. Monoclonal antibodyrecognized chondroitin sulfate $A$ was effectively used to treat ameloblastoma. Selenium-chondroitin sulfate nanoparticles demonstrated positive effects in therapy of Kashin-Beck disease (KBD) and osteoarthritis
\end{abstract}

Received 18th July 2019

Accepted 10th August 2019

DOI: $10.1039 / \mathrm{c} 9 \mathrm{ra05546 \textrm {k }}$

rsc.li/rsc-advances proteoglycan synthesis and also inhibits the formation of proteolytic enzymes and nitric oxide. Chondroitin sulphate is a double glycone moiety formed by glycosidic linkages with $\mathrm{N}$ acetylgalactosamine and D-glucuronic acid. ${ }^{11}$

\section{Synthesis of chondroitin sulphate and its modifications}

Various enzymes, such as $\mathrm{N}$-acetylgalactosamine transferase, Dglucuronic acid transferases, chondroitin synthases, CS $\mathrm{N}$-acetylgalactosaminyl transferases (I \& II), CS glucuronyl transferase and CS polymerization factor, catalyze proper co-ordination during chain elongation by phosphorylation or sulfation reactions; however, no single enzyme can develop the total chondroitin sulphate chain. ${ }^{\mathbf{1 2 , 1 3}}$ Another set of enzymes, such as chondroitin-4-sulfotransferase, chondroitin-6-sulfotransferase or GalNAc-4 sulfate 6-O-sulfotransferase, are necessary for sulfation reactions in $\mathrm{N}$-acetylgalactosamine residues, respectively. ${ }^{\mathbf{1 4 , 1 5}}$

\section{Pharmaceutical applications of chondroitin}

3.1 Decorated chondroitin sulfate nanoplatinum for the treatment of osteoarthritis

Biofabrication of platinum nanoparticles (PtNPs) using chondroitin sulfate was developed using a facile, eco-friendly route by heating leaf extract and $\mathrm{H}_{2} \mathrm{PtCl}_{6} \cdot 6 \mathrm{H}_{2} \mathrm{O}$ (chloroplatinic acid) solution to produce a brown PtNPs dispersion. Transmission electron microscopy (TEM), energy dispersive spectroscopy (EDX), X-ray diffraction (XRD), Fourier transform infrared
${ }^{a}$ Department of Pharmaceutical Sciences, Guru Ghasidas Vishwavidyalaya (A Central University), Bilaspur-495009, C.G., India. E-mail: drdilip71@gmail.com; Tel: +91-7389263761

${ }^{b}$ School of Pharmaceutical Sciences and Technology, Sardar Bhagwan Singh University, Dehradun-248161, Uttarakhand, India 
(FTIR) spectroscopy and selected area electron diffraction (SAED) were used to analyze the synthesized PtNPs. TEM analysis showed that the PtNPs had irregular shapes within the size range from 3 to $5 \mathrm{~nm}$. Zeta potential studies revealed that the surface charge of the synthesized PtNPs was negative $(-25.6$ $\mathrm{mV}$ ). FTIR analysis and zeta potential measurements confirmed that chondroitin sulfate was capped onto the surface of the nanoparticles. XRD and SAED data confirmed the crystalline nature of the synthesized nanoparticles (Fig. 1). In vitro cytotoxicity data showed the biocompatibility of PtNPs against osteoarthritis chondrocytes, which confirmed that the obtained nanoparticles may potentiate the treatment of osteoarthritis. ${ }^{\mathbf{1 6}}$

\subsection{Strontium chondroitin sulfate and the evaluation of its capability to attenuate osteoarthritis}

Strontium chondroitin sulfate (SrCS), a new polysaccharidemetal ion complex, consists of a combination of chondroitin sulfate and strontium, which are widely used in the treatment of osteoarthritis. The clinical potential of SrCS in osteoarthritis treatment was evaluated by cell proliferation tests, the reverse transcriptase-polymerase chain reaction (RT-PCR) and preliminary animal studies. The material characterization results verified that strontium was successfully embedded into chondroitin sulphate through the replacement of sodium in the original structure, which helped to form a new polysaccharidemetal ion complex. Cell proliferation results confirmed that SrCS has excellent biocompatibility for chondrocytes and osteoblasts. The RT-PCR results revealed that SrCS can significantly increase the expression of COLII and ACAN and decrease that of MMP1 and MMP13 in chondrocytes, and it also decreases IL-6 and IL-1 in both chondrocytes and osteoblasts. Preliminary animal studies demonstrated that SrCS can effectively simulate articular cartilage formation in SD rats after modified Hulth's osteoarthritis modeling surgery (Fig. 2). Therefore, SrCS may be an effective chemical for osteoarthritis clinical management and may also be beneficial for various biomaterials in cartilage tissue engineering. ${ }^{17}$

\subsection{Chondroitin sulfate from Scophthalmus maximus for treating osteoarthritis}

Chondroitin sulfate obtained from Scophthalmus maximus shark was observed to have good effects on osteoarthritis in the clinic. Scophthalmus maximus is an important source to provide chondroitin sulphate for the treatment of OA. A similar therapeutic effect was observed for CS from Scophthalmus maximus (CS-SM) for treating osteoarthritis in rats. Intragastric

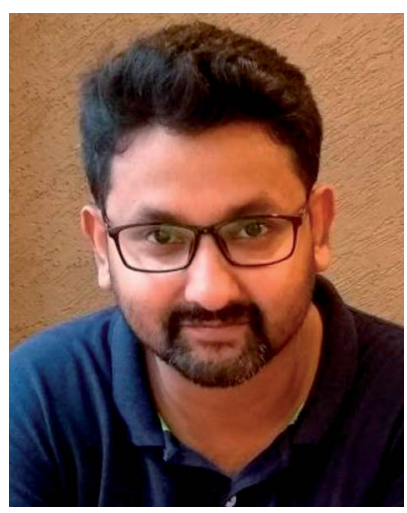

Supriyo Saha, PhD, MPharm, is an Assistant Professor in the School of Pharmaceutical Sciences and Technology at Sardar Bhagwan Singh University, Dehradun, India. His areas of research interest include synthetic chemistry and polymer science. He has published many research papers in peer-reviewed national and international scientific journals. He has written book chapters and has written one book. Dr Saha, in his nine years of research and teaching, has worked as a reviewer for many scientific publications. research interests include isolation, structure elucidation and biological evaluation of indigenous plants and natural biopolymers. He has published 164 full research papers in reputed peerreviewed national and international scientific journals with good impact factors, and he has contributed 113 abstracts to various national and international conferences. He has written one book and twenty-six book chapters that were published by reputed international publishers. His research publications have acquired a highly remarkable cited record in Scopus and Google Scholar $(\mathrm{H}-$ index 35; i-10-index 82; total citations 3722 to date). Dr Pal, in his 20 years in the research-oriented teaching profession, has also received 13 prestigious national and international professional awards. He has guided the dissertation theses of $7 \mathrm{PhD}$ and 39 Master's students. He is a reviewer and Editorial Board member for 27 and 29 scientific journals, respectively. Dr Pal is also the Editor-in-Chief of one good research journal. He is a member and life member of fifteen professional organizations. 


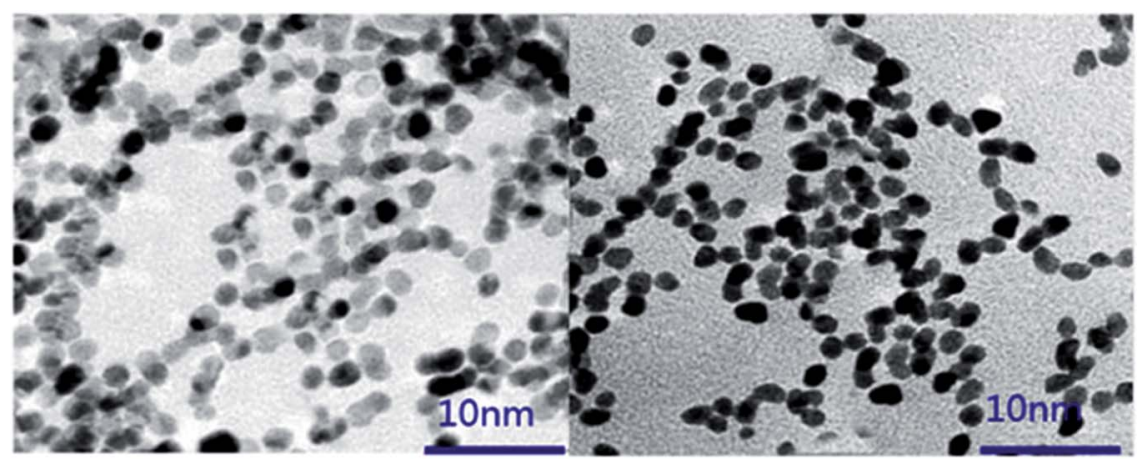

Fig. 1 TEM image of PtNPs synthesized using chondroitin sulfate. [Source: Yin et al., ${ }^{16}$ Copyright@ 2017 with permission from Elsevier B.V.]

administration of CS-SM revealed that CS-SM could function in articular cartilage in OA by inhibiting the degradation of cartilage, decreasing the apoptosis of chondrocytes, decreasing the amounts of interleukin-1, tumor necrosis factor- $\alpha$ and prostaglandins E2 in synovial fluid (Table 1), downregulating the protein expression of matrix metalloproteinase- 1 and upregulating the protein expression of tissue inhibitor of metalloproteinase-1 (Fig. 3). These outcomes suggest that oral chondroitin sulphate from Scophthalmus maximus is a new potential source of therapy for OA. ${ }^{18}$
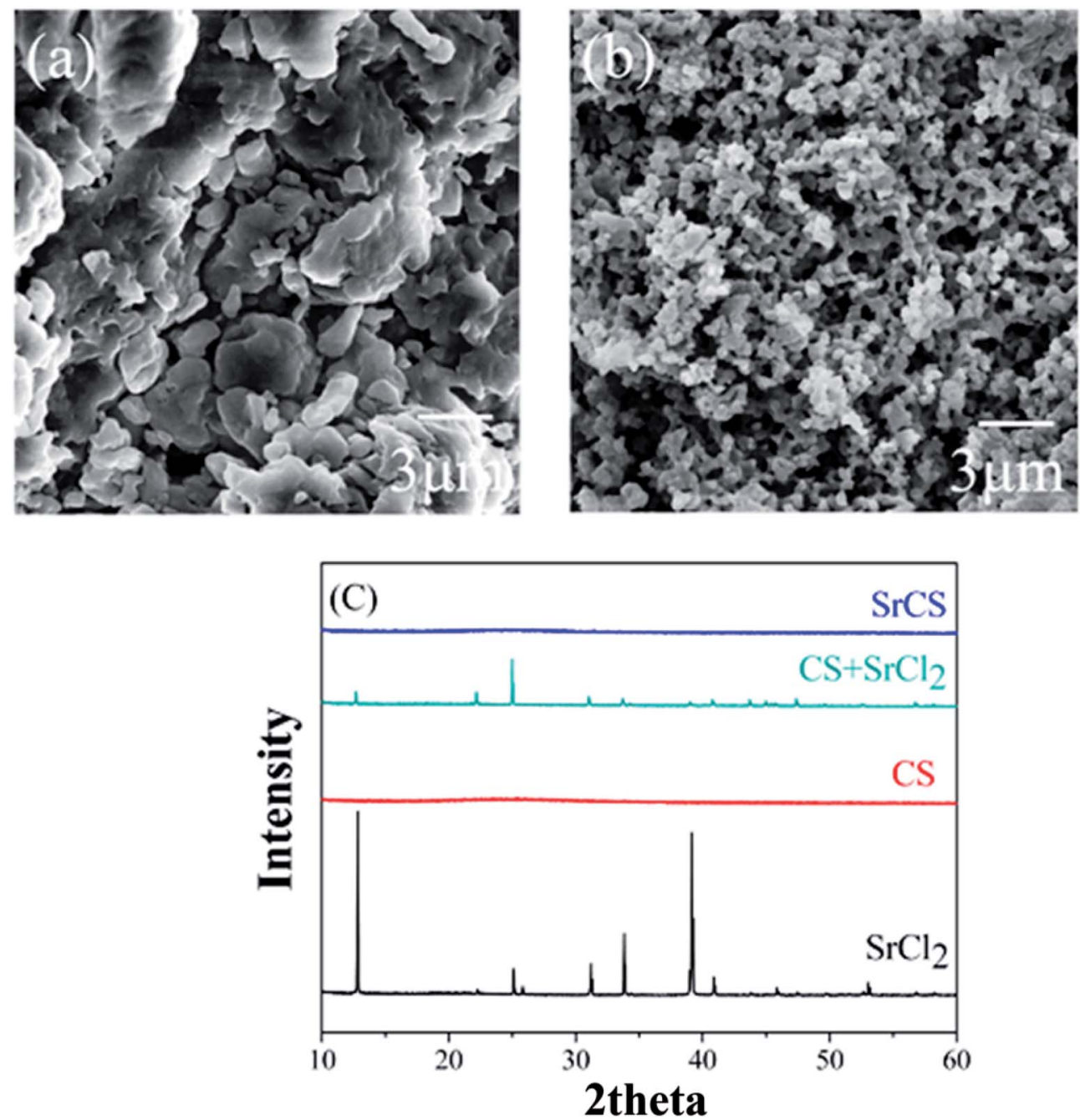

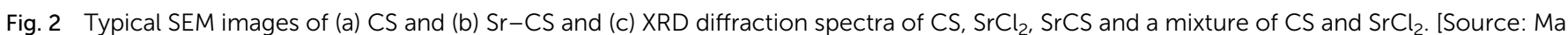
et al., ${ }^{17}$ Copyright@ 2017 with permission from Elsevier B.V.] 


\subsection{Anticoagulant activities of fucosylated chondroitin sulfates from Holothuria scabra}

A new generation fucosylated chondroitin sulfate (HsG) with a molecular weight of $69.1 \mathrm{kDa}$ was obtained from the sea cucumber Holothuria scabra. Monosaccharide composition analysis, disaccharide composition analysis, IR, ${ }^{1} \mathrm{H}$ and ${ }^{13} \mathrm{C}$ NMR spectra, and methylation analysis were used to investigate the structure of HsG. The methylation results of desulfated/ carboxyl-reduced polysaccharides and the analysis of unsaturated disaccharides generated through the enzymolysis of the defucosed polysaccharides showed that each branch contains one fucopyranosyl residue, wherein $55.7 \%$ of the fucopyranosyl residues are linked to the O-6 position of the $\mathrm{N}$-acetylgalactosamine moiety, $21.2 \%$ of the fucopyranosyl residues are linked to the $\mathrm{O}-3$ position of $\beta$-D-glucuronic acid, $13.0 \%$ of the fucopyranosyl residues are linked to the O-4 positions of the $\mathrm{N}$-acetylgalactosamine moiety, and $10.1 \%$ of the fucopyranosyl residues are not linked to sulfate groups on the backbone. The backbone $\rightarrow 4)$ GlcUA $\beta(1 \rightarrow 3)$ GalNAc $\beta(1 \rightarrow$ and the sulfated fucose branches were composed of $\beta$-D-glucuronic acid, $N$ acetyl- $\beta$-D-galactosamine, $\alpha$-L-fucose and sulfate groups in a molar ratio of $1: 1.72: 2.34: 3.29$. The anticoagulant activities of HsG were estimated and compared with those of heparin. The outcomes revealed that HsG prolonged the activated partial thromboplastin time. ${ }^{19}$

\subsection{Two fucosylated chondroitin sulfates from the sea cucumber Eupentacta fraudatrix}

Two fucosylated chondroitin sulfates, EF1 and EF2, were obtained from the sea cucumber Eupentacta fraudatrix, and anionexchange chromatography on DEAE-Sephacel was used to separate the polysaccharides by elution with $0.75 \mathrm{M}$ and $1.0 \mathrm{M}$ $\mathrm{NaCl}$ solutions. Chemical and NMR spectroscopic methods were used to identify the structure of the biopolymers. The backbone of EF1 was composed of chondroitin sulfate A and E units in a ratio of about $1: 1$. The core of EF2 along with chondroitin sulfate $\mathrm{A}$ and $\mathrm{E}$ fragments contained the unusual disaccharide repeating units $\rightarrow 4)-\beta$-D-GlcpA2S3S- $(1 \rightarrow 3)-\beta-$ D- $^{-}$ GalpNAc6S- $(1 \rightarrow$. The main type of branch in both polysaccharides was an $\alpha$-L-Fucp3S4S unit attached to O-3 of GlcA residues, and another type of branch was found to be the disaccharide fragment $\alpha$-L-Fucp- $(1 \rightarrow 2)-\alpha$-L-Fucp3S4S- $(1 \rightarrow$ linked to O-3 of GlcA. The presence of structurally different

Table 1 The levels of IL-1 $\beta$, TNF- $\alpha$ and PGE2 in synovial fluid (mean \pm standard deviation, $n=8$ ). [Source: Zhenkun et al., ${ }^{18}$ Copyright $@ 2017$ with permission from Elsevier B.V.] ${ }^{a}$

\begin{tabular}{llcl}
\hline Group & IL-1 $\beta / \mathrm{pg} \mathrm{mL}^{-1}$ & TNF- $\alpha / \mathrm{pg} \mathrm{mL}$ & PGE2/pg mL \\
\hline Control & $13.05 \pm 0.129^{* * \# \#}$ & $6.58 \pm 0.071^{* * \# \#}$ & $0.46 \pm 0.017^{* * \# \#}$ \\
Sham & $13.38 \pm 0.080^{* * \# \#}$ & $7.39 \pm 0.055^{* * \# \#}$ & $0.45 \pm 0.025^{* * \# \#}$ \\
OA & $62.78 \pm 0.631^{\# \#}$ & $23.31 \pm 0.403^{\# \#}$ & $5.63 \pm 0.125^{\# \#}$ \\
CS-SM & $33.64 \pm 0.407^{* *}$ & $13.63 \pm 0.380^{* *}$ & $2.28 \pm 0.050^{* *}$ \\
CS-S & $34.94 \pm 0.410^{* *}$ & $13.23 \pm 0.484^{* *}$ & $2.22 \pm 0.062^{* *}$
\end{tabular}

${ }^{a}$ Compared to OA group $={ }^{*} P<0.05,{ }^{*} P<0.01$; compared to CS-S group $={ }^{\#} P<0.05,{ }^{\# \#} P<0.01$. fucosylated chondroitin sulfates in one species of sea cucumber is rather unusual. ${ }^{20}$

\subsection{A silk fibroin-chondroitin sulfate scaffold with immuno- inhibition properties for articular cartilage repair}

Chondroitin sulfate (CS) and silk fibroin show excellent biocompatibility as tissue engineering scaffolds. The rapid degradation rate of pure CS scaffolds afforded effective neotissue that is similar to natural articular cartilage. Due to its mechanical properties, long-lasting in vivo stability and hypoimmunity, silk fibroin was used as a structural constituent material. The combination of silk fibroin and CS synergistically promoted articular cartilage defect repair. Silk fibroin (silk) and silk fibroin/CS (silk-CS) scaffolds were also fabricated with saltleaching, freeze-drying and crosslinking methodologies. In vitro cell adhesion, proliferation and migration with human articular chondrocytes were used to investigate the biocompatibility of the scaffolds. The silk-CS scaffold maintained a better chondrocyte phenotype than the silk scaffold; moreover, the silk-CS scaffold decreased the chondrocyte inflammatory response induced by interleukin (IL)-1. After 6 and 12 weeks of implantation, in ICRS (International Cartilage Repair Society) histological evaluations, the rabbit osteochondral defect model was used to evaluate in vivo cartilage repair. The silk-CS scaffold induced more neo-tissue formation and better structural restoration than the silk scaffold. Therefore, the silk fibroin/ chondroitin sulfate scaffold exhibited immuno-inhibition properties for cartilage tissue engineering. ${ }^{21}$

\subsection{Effects of fucosylated CS from M. magnum on coagulation}

A fucosylated CS MM was obtained from the sea cucumber Massinium magnum. Chemical and NMR spectroscopic methods were used to evaluate the structure of this polysaccharide. The backbone of MM was constituted by small amounts of chondroitin sulphate. The fucosylated chondroitin sulphate extracted from $M$. magnum was observed to have anticoagulation properties, with greater inhibition of thrombin than of heparin. ${ }^{22}$

\subsection{Anticoagulant properties of fucosylated chondroitin sulfate isolated from Holothuria mexicana}

Fucosylated chondroitin sulfate (HmG) was obtained from the sea cucumber Holothuria mexicana. Monosaccharide composition, disaccharide composition, IR, ${ }^{1} \mathrm{H}$ and ${ }^{13} \mathrm{C}$ NMR spectroscopy, and two-dimensional NMR spectroscopy studies of degraded HmG (DHmG) were used to characterize its structure. The backbone of $\mathrm{HmG}$ was identified as chondroitin 6-O sulfate, while the major O-4 sulfated fucose branched at the O-3 position of glucuronic acid in almost every disaccharide unit. The anticoagulant activities of HmG and DHmG were examined and compared with those of heparin and low molecular weight heparin. The outcomes indicated that HmG and DHmG both significantly prolonged the activated partial thromboplastin time, and these properties were highly related to their molecular weights (Table 2). DHmG showed similar anticoagulant 


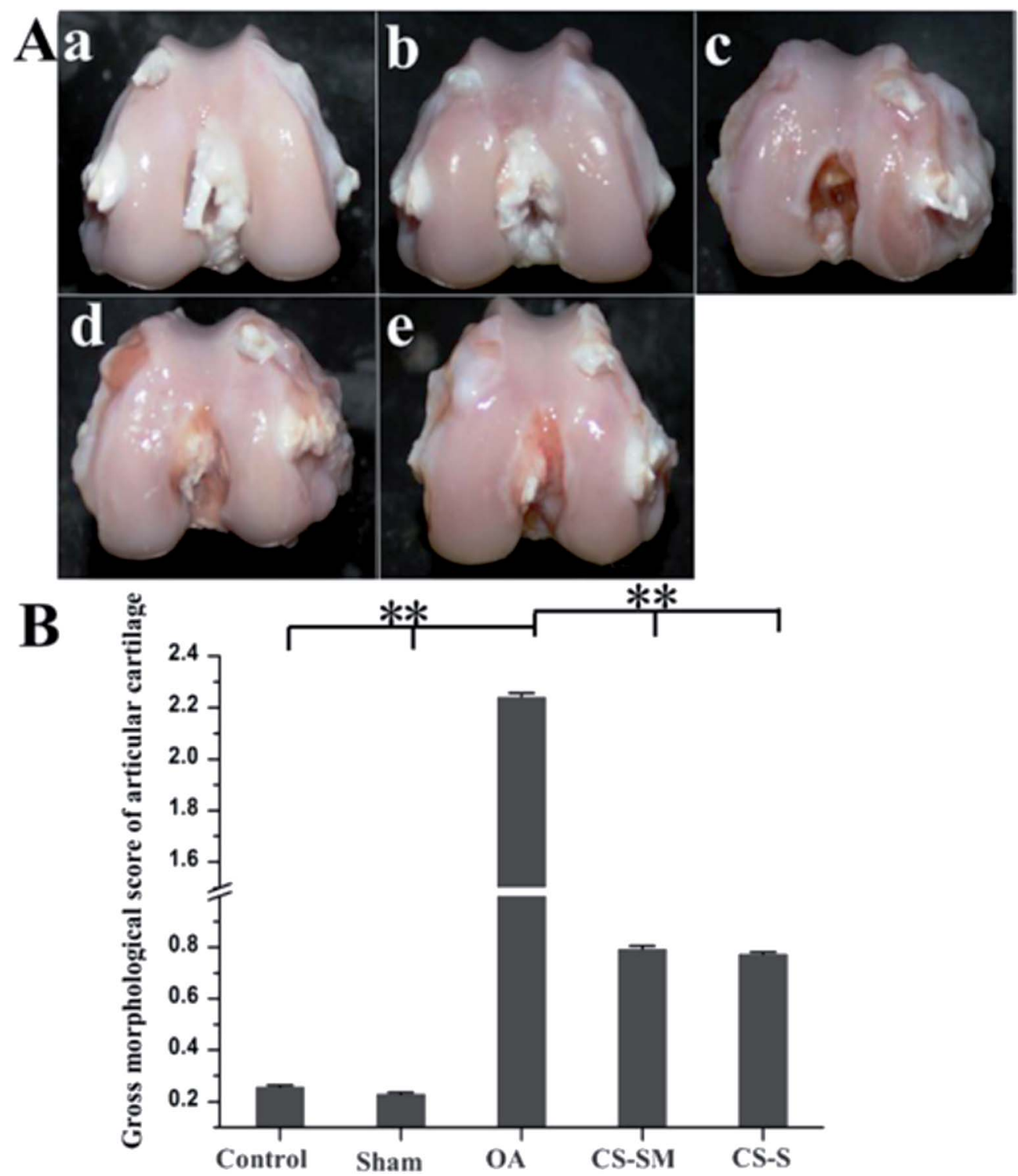

Fig. 3 Articular morphology observation. (A) Macroscopic morphology evaluation of articular cartilage: (a) control group, (b) sham group, (c) OA group, (d) CS-SM-treated group, (e) CS-S-treated group. (B) Gross morphological scores of articular cartilage (**P<0.01; $n=8$ for each group). [Source: Zhenkun et al., ${ }^{18}$ Copyright@ 2017 with permission from Elsevier B.V.]

properties to low molecular weight heparin with less bleeding risk; thus, it is a safer anticoagulant drug. ${ }^{23}$

\subsection{CS-enriched contact lenses}

Hemoderivative tears are used to maintain ocular surface homeostasis. Platelet lysate-loaded contact lenses have shown

Table 2 Physicochemical characteristics of intact $\mathrm{HmG}$ and purified low-molecular-weight DHmG. [Source: Jiaojiao et al., ${ }^{23}$ Copyright $\odot$ 2017 with permission from Elsevier B.V.]

\begin{tabular}{llllll}
\hline & \multicolumn{5}{c}{ Molar ratios } \\
\cline { 3 - 6 } Fraction & $M_{\mathrm{w}}(\mathrm{kDa})$ & GalNAc & GlcA & Fuc & Sulfate \\
\hline HmG & 99.8 & 1.00 & 0.93 & 1.40 & 3.24 \\
DHmGa & 11.6 & 1.00 & 0.84 & 1.29 & 3.05
\end{tabular}

greater efficiency to treat cornea infection. CS is associated with platelet lysate. Also, CS reacts with basic fibroblast growth factor, insulin-like growth factor, vascular endothelial growth factor, platelet-derived growth factor and transforming growth factor beta via electrostatic interactions for stability and decreased degradation in solution. CS-loaded contact lenses with or without platelet lysate were compared for the release of growth factors into corneal surface lesions. PureVision 1, a specialized contact lense, revealed good in vitro proliferation of corneal cells and facilitated cut closure in corneal constructions. $^{24}$

\subsection{Effects of chondroitin sulfate on siRNA biodistribution and gene silencing in mice}

Intravenous injection of chondroitin sulfate (CS) followed by intravenous injection of siRNA/cationic liposome complexes 
(siRNA lipoplexes) was used to deliver siRNAs to the liver and suppress the expression of target genes. The effects of the injection order of CS and siRNA lipoplexes on the biodistribution of siRNA and gene silencing in the liver after sequential injection were examined. After intravenous injection of siRNA lipoplexes into mice, the siRNA largely accumulated in the lungs. Injection of siRNA lipoplexes followed by injection of CS decreased siRNA accumulation in the lungs and increased it in the liver. Also, agglutinates of erythrocytes caused by the addition of siRNA lipoplexes were redispersed by the addition of CS; this indicates that the agglutinates that accumulated in the lungs after injection of siRNA lipoplexes were split up by CS injection. Also, injection of apolipoprotein B (ApoB) siRNA lipoplexes followed by injection of CS did not suppress ApoB mRNA levels in the liver (Fig. 4). These outcomes confirm that the injection order of CS and siRNA lipoplexes is important for gene silencing effects in the liver, although the sequential injection delivered siRNA efficiently to the liver. ${ }^{25}$

\subsection{Fucosylated chondroitin sulfate from Holothuria mexicana and its effects on growth factor binding and anticoagulation}

A structurally distinct glycosaminoglycan, fucosylated chondroitin sulfate (FCS), was isolated from the body wall of sea cucumber; it possesses many biological properties and pharmacology functions. NMR spectroscopy and hydrophilic liquid interaction chromatography with Fourier transform mass spectrometry (HILIC-FTMS) were used to characterize the refined structure of FCS isolated from the sea cucumber Holothuria mexicana (FCShm); four types of branches were observed in FCShm. Among these, two branches were $\alpha$-L-Fuc-2S4S (where Fuc is fucose and $S$ is sulfo) and $\alpha$-L-Fuc-4S linked to O-3 of glucuronic acid residues, while others were identified as $\alpha$-L-Fuc-4S and $\alpha$-L-Fuc-3S4S attached to O-6 of $N$-acetylgalactosamine residues. Furthermore, the fucosyl branches were $\alpha$-1,3-linked with different degrees of polymerization from 1 to 5. FCShm exhibited high affinity towards fibroblast growth factors 1 and 2, which are involved in neo-vascularization. Furthermore, FCShm displayed intrinsic anticoagulant activity and inhibited thrombin and factor $\mathrm{Xa}$ activation by antithrombin III. These results indicate that a novel structural FCS was developed with anti-angiogenesis and anticoagulation properties. $^{26}$

\subsection{Antiproliferative properties of chondroitin sulfate/ dermatan sulfate from tunisian fish skins}

Chondroitin sulfate/dermatan sulfate GAGs were isolated from the skins of grey triggerfish (GTSG) and smooth hound (SHSG). Treatment with chondroitinase $\mathrm{ABC}$ over the disaccharide composition showed the presence of a nonsulfated disaccharide, monosulfated disaccharides and disulfated disaccharides in different percentages. The amounts of nonsulfated disaccharide, named $\Delta$ DioS, of GTSG and SHSG were estimated to be $3.5 \%$ and $5.5 \%$, respectively, while the amounts of monosulfated disaccharides, named $\Delta \mathrm{Di} 6 \mathrm{~S}$ and $\Delta \mathrm{Di} 4 \mathrm{~S}$, were estimated to be $18.2 \%$ and $59 \%$ and $14.6 \%$ and $47.0 \%$, respectively.
Capillary electrophoresis analysis data of GTSG and SHSG showed $99.2 \%$ and $95.4 \%$ chondroitin sulfate/dermatan sulfate, respectively. Polyacrylamide gel electrophoretic analysis showed that GTSG and SHSG have molecular masses of 41.72 $\mathrm{kDa}$ and $23.8 \mathrm{kDa}$, respectively. At $200 \mu \mathrm{g} \mathrm{mL} \mathrm{m}^{-1}$ doses of GTSG and SHSG, HCT116 cell proliferation was inhibited $(p<0.05)$ by $70.6 \%$ and $72.65 \%$, respectively. Both GTSG and SHSG demonstrated potential antiproliferative behavior. ${ }^{27}$

\subsection{Chondroitin-4-sulfate polysaccharide as a sulfated polysaccharide by hexacyanoferrate(III) in alkaline solutions with the synthesis of a novel coordination biopolymer chelating agent}

The reduction of hexacyanoferrate (III) by biodegradable chondroitin-4-sulfate (CS) in alkaline solution at a constant ionic strength of $1.0 \mathrm{~mol} \mathrm{dm}{ }^{-3}$ was investigated spectrophotometrically. The kinetic results confirmed first-order dependence for the oxidant and fractional-order kinetics with respect to CS. The influence of base on the oxidation rate indicated that the reaction was base-catalyzed. Kinetic evidence for the formation of a $1: 1$ intermediate complex was revealed. ${ }^{28}$

\subsection{Effects of chondroitin sulphate on synovitis in knee osteoarthritic patients}

The effects of chondroitin sulfate (CS) on synovitis in patients with knee osteoarthritis (KOA) was estimated by ultrasonography. A randomized, single-blind, controlled trial was developed with 70 patients with primary KOA, who were treated for 6 months with CS or acetaminophen. After evaluation of KOA at baseline, 6 weeks, 3 months and 6 months, ultrasonography was used to assess synovitis (following the Outcome Measures in Rheumatology (OMERACT) expertise group definition), the visual analogue scale and Lequesne index were used to measure pain and function, and ELISA was used to quantify inflammatory mediators in serum and synovial fluid. The results confirmed that presence of synovitis was decreased by $50 \%$ in the CS group, while a $123 \%$ increase was observed in the ACT arthritis group. Moreover, patients without initial synovitis who were treated with ACT reached $85.71 \%$ synovitis onset, but only $25 \%$ onset was observed in the CS group. Both therapies showed improved articular function, but only CS resulted in significant pain improvement at the end of the treatment. Changes in Regulated on Activation Normal T Cell Expressed and Secreted (RANTES) concentration and UCN (urocortin) synovial fluid concentration were associated with CS treatment. Treatment with CS directly prevented synovitis onset or decreased its presence and also decreased KOA symptoms. Antiinflammatory effects of CS were observed by changes in the RANTES and UCN concentrations. ${ }^{29}$

\subsection{Anti-diabetic effects of chondroitin sulfate on normal and type 2 diabetic mice}

Chondroitin sulphate with different molecular weights (from 170000 to $10000 \mathrm{Da}$ ) showed in vitro inhibitory effects on $\alpha$ glucosidase activity. Higher inhibition of $\alpha$-glucosidase activity was demonstrated by low-molecular-weight chondroitin 


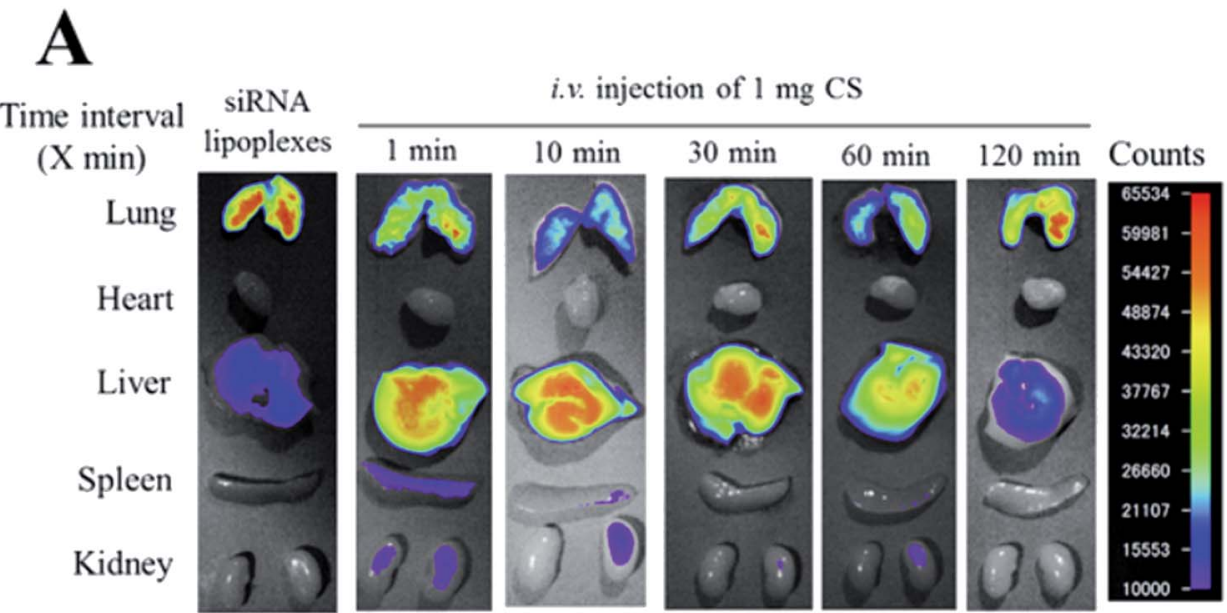

B

Time interval (X min)

$1 \min$

i.m. injection of $10 \mathrm{mg} \mathrm{CS}$

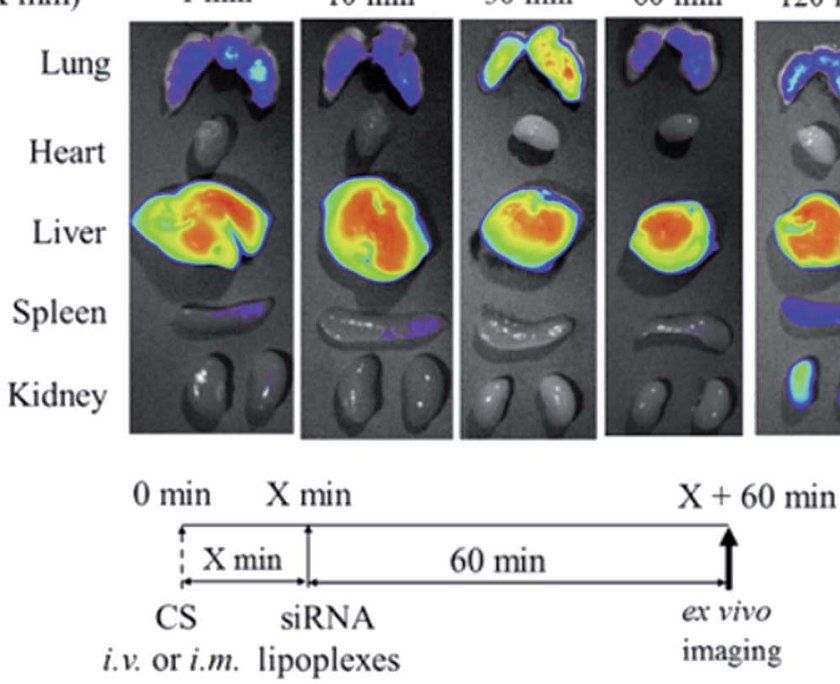

Fig. 4 Effect of chondroitin sulfate (CS) on the biodistribution of small interfering RNA (siRNA) in mice after sequential injection of CS plus siRNA lipoplexes. At 1, 10, 30, 60, or 120 min after intravenous injection of $1 \mathrm{mg}$ CS (A) or after intramuscular injection of $10 \mathrm{mg}$ CS (B), lipoplexes with $50 \mathrm{mg} \mathrm{Cy5.5-siRNA}$ were administered intravenously to mice. Ex vivo images of dissected tissues were obtained at 60 min after sequential injection. i.v.; intravenous injection, i.m.; intramuscular injection. [Source: Hattori et al., ${ }^{16}$ copyright@ 2017 with permission from Elsevier B.V.]

sulphate $(\mathrm{MW}<16000 \mathrm{Da})$ than by high-molecular-weight chondroitin sulphate. Elevation of postprandial blood glucose in mice loaded with sucrose or starch was inhibited by lowmolecular-weight chondroitin sulphate. The elevation of fasting blood glucose was inhibited in the CS group (Table 3). The outcomes revealed that low molecular weight chondroitin sulphate prevents the elevation of postprandial blood glucose levels with minimization of carbohydrate digestion in the gastrointestinal tract. ${ }^{30}$

\subsection{Inhibitory effects of chondroitin sulfate oligosaccharides on bovine testicular hyaluronidase}

The extracellular matrices of animal tissues are composed of hyaluronan and chondroitin sulfates as principal components. Hyaluronan and chondroitin sulfate oligosaccharides showed inhibitory effects on the hydrolysis and transglycosylation reactions of bovine testicular hyaluronidase using hyaluronan as a substrate. HPLC was used to analyze the hydrolysis and transglycosylation activities of the oligosaccharides. Chondroitin sulfate oligosaccharide showed dose-dependent

Table 3 Hydrolysis conditions, molecular weights and degrees of sulfation of hydrolyzed chondroitin sulfate. [Source: Moto et al., Copyright@ 2018 with permission from Elsevier B.V.]

\begin{tabular}{lll}
\hline Hydrolysis conditions & $\begin{array}{l}\text { Molecular weight } \\
(\mathrm{Da})\end{array}$ & $\begin{array}{l}\text { Degree of sulfation } \\
(\%)\end{array}$ \\
\hline Not hydrolyzed & 170000 & 19.1 \\
$80^{\circ} \mathrm{C}, 20 \mathrm{~h}$ & 130000 & 19.1 \\
$90^{\circ} \mathrm{C}, 20 \mathrm{~h}$ & 82000 & 19.1 \\
$100{ }^{\circ} \mathrm{C}, 14 \mathrm{~h}$ & 50000 & 19.0 \\
$100^{\circ} \mathrm{C}, 24 \mathrm{~h}$ & 33000 & 18.8 \\
$110^{\circ} \mathrm{C}, 24 \mathrm{~h}$ & 16000 & 18.2 \\
$120^{\circ} \mathrm{C}, 16 \mathrm{~h}$ & 10000 & 17.1
\end{tabular}


inhibition of bovine testicular hyaluronidase; however, hyaluronan and chondroitin oligosaccharides did not. ${ }^{31}$

\subsection{Antifouling properties of polyurethane films modified by chondroitin sulfate}

Polyurethane-chondroitin sulfate (PU-CS) film was developed using chemically grafted $N$-boc-1,3-propanediamine as an antifouling agent. Different mass fractions of $N$-boc-1,3propanediamine afforded PU-CS films with different CS grafting densities. The surface properties of the PU-CS films were characterized by studying the surface properties, protein adsorption and glycosaminoglycan adhesion behavior of the films. Furthermore, the highest CS grafting density of $3.70 \mu \mathrm{g}$ $\mathrm{cm}^{-2}$ was observed on the film. The outcomes revealed that the CS grafting density improved the hydrophilicity and antifouling performance of the films. Adsorption of fibrinogen (BFG), human serum albumin (HSA) and lysozymes (LYS) decreased by $81.4 \%, 95.0 \%$ and $76.5 \%$, respectively, and the adhesion of chondroitin (CS), heparin (HP) and hyaluronic acid (HA) decreased by $70.6 \%, 87.4 \%$ and $81.3 \%$, respectively in the best antifouling film. Furthermore, the co-adsorption of proteins and glycosaminoglycans decreased by up to $86.9 \%$ and $75.5 \%$, respectively. Co-adsorption of proteins and glycosaminoglycans on PU-CS indicated that the proteins promoted inorganic salt deposition, while the glycosaminoglycans inhibited crystal growth. Negatively charged polysaccharides increased the formation of smaller crystals; this was used as a theoretical and practical guide to develop novel anti-encrusted urinary stents. ${ }^{32}$

\subsection{Hexagonal-shaped chondroitin sulfate self-assemblies showed high anti-HSV-2 activity}

Biomimetic supramolecular hexagonal-shaped nanoassemblies composed of chondroitin sulfate were developed as anti-HSV-2 agents and compared against native chondroitin sulfate. Hydrophobically modified chondroitin sulfate and cyclodextrin were mixed in water to form nanoassemblies. The alkyl chain length was optimized on chondroitin sulfate, and optimizing the ratio between hydrophobically-modified chondroitin sulfate and cyclodextrin afforded more cohesive and well-structured nanoassemblies with higher cyclodextrin concentrations and longer alkyl chain lengths. Moreover, the hexagonal nanoassemblies showed better antiviral activity against HSV-2 in comparison with that of hydrophobically-modified chondroitin sulfate. This result confirmed that a new biomimetic formulation was developed to target the HSV-2 viral strain. ${ }^{33}$

\subsection{Structural modulation of gut microbiota by chondroitin sulfate and its oligosaccharide}

In vitro degradation and fermentation of chondroitin sulfate (CS) is performed by specific human gut microbes, which suggests a possible way to potentiate the in vivo effects of chondroitin sulfate on gut microbiota composition. Chondroitin sulfate and chondroitin sulfate oligosaccharide (CSO) were modulated in the gut microbiota in Kunming mice by high-throughput sequencing. Heatmap and principal component analysis (PCA) suggested that female microbiota are more susceptible than male microbiota towards CS and CSO treatment. CS and CSO created diversified effects on the abundance of Bacteroides S24-7, Helicobacter, Odoribacter, Prevotellaceae and Lactobacillus in male mice versus female mice. This collective information demonstrated that sex-dependent effects were produced on gut microbiota by CS and CSO. ${ }^{34}$

\subsection{Chondroitin sulfate proteoglycans from salmon nasal cartilage inhibit angiogenesis}

Because cartilage lacks nerves, blood vessels, and lymphatic vessels, it is thought to contain factors that inhibit the growth and development of those tissues. Chondroitin sulfate proteoglycans (CSPGs) are the principal extracellular component found in cartilage; they contribute to joint flexibility. They also regulate extracellular signaling due to the presence of glycosaminoglycans and CS. CS and CSPG inhibit the regeneration of the axonal membrane. Salmon nasal cartilage proteoglycan (PG) was testified to inhibit angiogenesis. Salmon nasal cartilage proteoglycan (PG) is a member of the aggrecan family of CSPG, and treatment with salmon proteoglycan inhibited endothelial cell adhesion and in vitro tube formation. Chondroitin sulphate derived from salmon PG showed anti-angiogenic activity; however, the core protein did not. Salmon PG was also found to help decrease matrix metalloproteinase enzyme expression and to inhibit the angiogenesis process in chick chorioallantoic membrane..$^{35}$

\subsection{Chondroitin sulfate attenuates formalin-induced persistent tactile allodynia}

In this study, chondroitin sulfate (CS) was used to help decrease virulence occurrence in osteoarthritis and joint pain; it was also effective against formalin-induced tactile allodynia in mice. After 10 days of formalin injection, oral repeated administration of CS (300 mg kg-1, b.i.d.) helped improve formalin-induced tactile allodynia. On the $14^{\text {th }}$ day after repeated administration of CS, spinal p38 MAPK was phosphorylated, which caused a subsequent increase in c-Fos-immunoreactive dorsal lumbar neurons. These outcomes suggested that CS improves formalininduced tactile allodynia through p38 MAPK phosphorylation inhibition and subsequent up-regulation of c-Fos expression in the dorsal lumbar spinal cord. ${ }^{36}$

\subsection{Potential involvement of chondroitin sulfate $A$ in the pathogenesis of ameloblastoma}

This study reflected the potential role of glycosaminoglycans against ameloblastoma pathogenesis. Ameloblastoma is a benign odontogenic tumor characterized by locally invasive behavior with high incidence of occurrence; meanwhile, glycosaminoglycans are structural components of cell membranes and the extracellular matrix. Immunohistochemical knowledge using monoclonal antibodies recognizing chondroitin sulfate A (CS-A), heparan sulfate (HS), and keratan sulfate (KS) was used to study formalin-fixed, paraffinembedded tissue sections of ameloblastoma, odontogenic keratocyst and dentigerous cyst. In the epithelial component and stroma of ameloblastoma, the levels of CS-A were significantly 
higher than in odontogenic keratocysts and dentigerous cysts. Furthermore, ameloblastoma contains more CS-A in stellate reticulum-like cells than in amelobast-like cells. Also, the concentrations of HS and KS were found to be higher in the epithelial component and lower in the stroma of ameloblastoma compared to CS-A. These outcomes revealed that CS-A is preferentially expressed in ameloblastoma compared to other odontogenic agents. ${ }^{37}$

\subsection{Antifouling properties of polyurethane films modified with chondroitin sulfate in urine}

The antifouling properties of urinary stent materials are greatly influenced by the composition and properties of the conditioning film. In this work, modified polyurethane films with chondroitin sulfate (PU-CS) with different grafting densities were developed to modify their anti-fouling properties. The effects of the composition and properties of the conditioning film on inorganic salt deposition and bacteria adhesion in urine greatly influenced the encrustation of urinary stents. The outcomes revealed that the roughness, water contact angle and zeta potential and the quantity of proteins and polysaccharides present in the conditioning films decreased with increasing CS grafting density of the polyurethane films. The highest bacterial inhibition rate was found for PU-CS with a CS grafting density of $3.70 \mathrm{~g} \mathrm{~cm}^{-2}$, and the least inorganic salt deposition was also observed amongst the PU-CSs in artificial urine. Amongst the formulations, PU-CS was observed to show less inorganic salt deposition than the other films. The presence of bacteria was not observed until the $21^{\text {st }}$ day in real urine with elevated $\mathrm{pH}$ values. These outcomes revealed that hydrophilicity, zeta potential and roughness were the important factors of the conditioning films for inorganic salt deposition and bacteria adhesion. Furthermore, the protein composition promoted the anti-encrustation properties, where encrustation was inhibited by polysaccharides. $^{38}$

\subsection{Antithrombotic activities of fucosylated chondroitin sulfates from two sea cucumbers}

A newer fucosylated chondroitin sulfate (FCS) derivative and its depolymerized fragment were isolated from the sea cucumber Cucumaria frondosa; this glycosaminoglycan is a promising antithrombotic and anticoagulant agent in vivo. The outcomes revealed that depolymerized FCS showed a better antithrombotic-hemorrhagic ratio than native fucosylated chondroitin sulfate on the electrically induced arterial thrombosis model in rats. Comparison data showed that the FCS extracted from sea cucumber possessed different sulfation patterns but similar antithrombotic effects. Therefore, the anticoagulation and antithrombosis properties of FCS are less dependent upon its sulfation pattern than on its molecular weight. These outcomes showed the importance of the structure-activity relationship of FCS to produce different alternative agents towards coagulation. ${ }^{39}$

\subsection{Altered expression of chondroitin sulfate structure- modifying sulfotransferases in articular cartilage from adult osteoarthritis and Kashin-Beck disease patients}

In this study, the expression behavior of enzymes which are involved in chondroitin sulfate (CS) sulfation in articular cartilage isolated from adult patients with osteoarthritis (OA) and Kashin-Beck disease (KBD) was studied using normal adults as controls. Articular cartilage samples were collected from normal, osteoarthritic and Kashin-Beck disease patients aged 38 to 60 years. Three groups were formed with six individual subjects in each group. The Safranin O staining method was used to examine the morphological and pathological grading of knee joint cartilage. Immunohistochemical staining and semi-quantitative analyses were used to analyze the localization and expression of enzymes involved in CS sulfation. CHST-3, CHST-11, CHST-12, CHST-13, CHST-15, UST, CHST-3, CHST-12, CHST-15, and UST showed lower positive staining rates for anabolic enzymes in the KBD and OA groups than in the control group. It was observed that the levels of CHST-11 and CHST-13 in the KBD group were different from those in the OA and control groups. Superficial and deep zones of KBD cartilage showed lower detected expression of all six CS sulfation enzymes compared to control and OA cartilage. In both $\mathrm{KBD}$ and $\mathrm{OA}$ adult patients, expression of CS structuremodifying sulfotransferase in the chondrocytes decreased, which greatly explained the cartilage damage and identified therapeutic targets for treatment. ${ }^{40}$

\subsection{Chondroitin sulfate immobilization at the surface of electrospun nanofiber meshes for cartilage tissue regeneration approaches}

Surface modification is a potential method to improve the mechanical properties, biocompatibility and surface bioactivity of biomaterial scaffolds; to confirm this, through $\mathrm{UV} / \mathrm{O}_{3}$ exposure and aminolysis, functionalized chondroitin sulfate (CS) was immobilized on the surface of electrospun poly(caprolactone) nanofiber meshes (PCL NFMs). CS-immobilization in PCL NFMs was characterized by contact angle, SEM, optical profilometry, FTIR, and X-ray photoelectron spectroscopy techniques. Furthermore, lower roughness and higher hydrophilicity were shown by CS-immobilized PCL NFMs than by samples without CS. Electrospun PCL NFMs with or without CS immobilization techniques were used to culture human articular chondrocytes (hACs). The results showed that hACs proliferated through the entire time course of the experiment in both types of nanofibrous scaffolds, as well as the production of glycosaminoglycans. Overexpression of cartilage-related genes such as aggrecan, collagen type II, COMP and Sox9 on both types of nanofibrous scaffolds was demonstrated by quantitative-PCR results. SEM and laser scanning confocal microscopy revealed the morphological behavior of the hACs; their characteristic round shapes and cellular agglomeration were observed exclusively on PCL NFMs with CS immobilization (Fig. 5). Therefore, immobilized CS on PCL NFMs provides a valid platform for cartilage tissue engineering. ${ }^{41}$ 


\subsection{Fucosylated chondroitin sulfates from the sea cucumbers Apostichopus japonicus and Actinopyga mauritiana}

Two fucosylated chondroitin sulfates (FCS) were isolated from the holothurian species Apostichopus japonicus (AJ) and Actinopyga mauritiana (AM), respectively; they were purified by ion exchange chromatography followed by gel filtration. Chemical and NMR spectroscopic methods were used to elucidate the structures of the biopolymers. A typical chondroitin core was built of repeating disaccharide units $\rightarrow 3)$ - $\beta$-D-GalNAc- $(1 \rightarrow 4)$ - $\beta$ D-GlcA- $(1 \rightarrow$ and decorated by sulfate groups and $\alpha$-L-Fuc branches. The two polysaccharides contained different patterns of sulfation of GalNAc and fucosyl branches, which were connected to O-3 of GlcA. Ratios of $2: 1$ and $1: 1$ were observed in GalNAc4S6S : GalNAc4S for AJ and AM, respectively. AJ was composed of Fucp2S4S and Fucp3S4S residues linked with O-3 of GlcA in a $3: 1$ ratio, while a $1: 4$ ratio was observed with AM. In both polysaccharides, small units of Fucp4S were attached to O-3 of GlcA. Furthermore, NMR spectral data confirmed the presence of Fucp3S residues linked to O-6 of GalNAc. ${ }^{42}$

\subsection{Selenium-chondroitin sulfate nanoparticles}

Ultrasonic and dialysis methods were used to synthesize a novel selenium-chondroitin sulfate (SeCS), and FTIR, XRD and TEM analytical data suggested that the SeCS nanoparticles underwent a self-aggregation progress in distilled water, with sizes between 30 and $200 \mathrm{~nm}$ and a selenium entrapment efficiency of about $10.1 \%$. In vitro MTT assay results demonstrated the antitoxin capacity of the SeCS nanoparticles. The SeCS nanoparticles were less cytotoxic to chondrocytes than sodium selenite. Therefore, SeCS showed increased T-2 toxin-induced chondrocyte apoptosis compared to chondroitin sulfate (Fig. 6). These outcomes indicated that the SeCS nanoparticles have exciting potential applications in therapy of Kashin-Beck disease (KBD) and osteoarthritis. ${ }^{43}$

\subsection{Hypercalcification of the organic matrix of bovine peritubular dentin}

Odontoblastic processes are responsible for secretion of the apatitic mineral dentin, which forms within the collagenous matrix (intertubular dentin, ITD). The interface between ITD and the process membrane was deposited with a highly calcified mineral (peritubular dentin, PTD); this helped to create a tubular system to penetrate the dentin, extending from the dentino-enamel junction to the predentin-dentin junction. ITD was studied by a laser capture technique, and SEM, TEM, and energy dispersive spectrometry (EDS) were used to analyze the PTD. The outcomes were subsequently compared with similar analyses of intact dentin slices containing ITD bounded-PTD annuli. Clearly marked boundaries between the ITD, PTD, and OP components were found by elemental line scans, and this was also correlated with differences in the composition and topographical surface roughness. The organic matrix of the PTD was sulfur rich, and an antibody labeling technique showed the sulfated organic component of chondroitin sulfate $\mathrm{B}$. The $\mathrm{S} / \mathrm{Ca}$ and $\mathrm{Ca} / \mathrm{P}$ ratios of the PTD organic matrix were distinctly higher than those of ITD; this indicated that the polysaccharide-bound $\mathrm{S}$ supplied anionic counterions and facilitated the formation of the apatitic PTD mineral. ${ }^{44}$
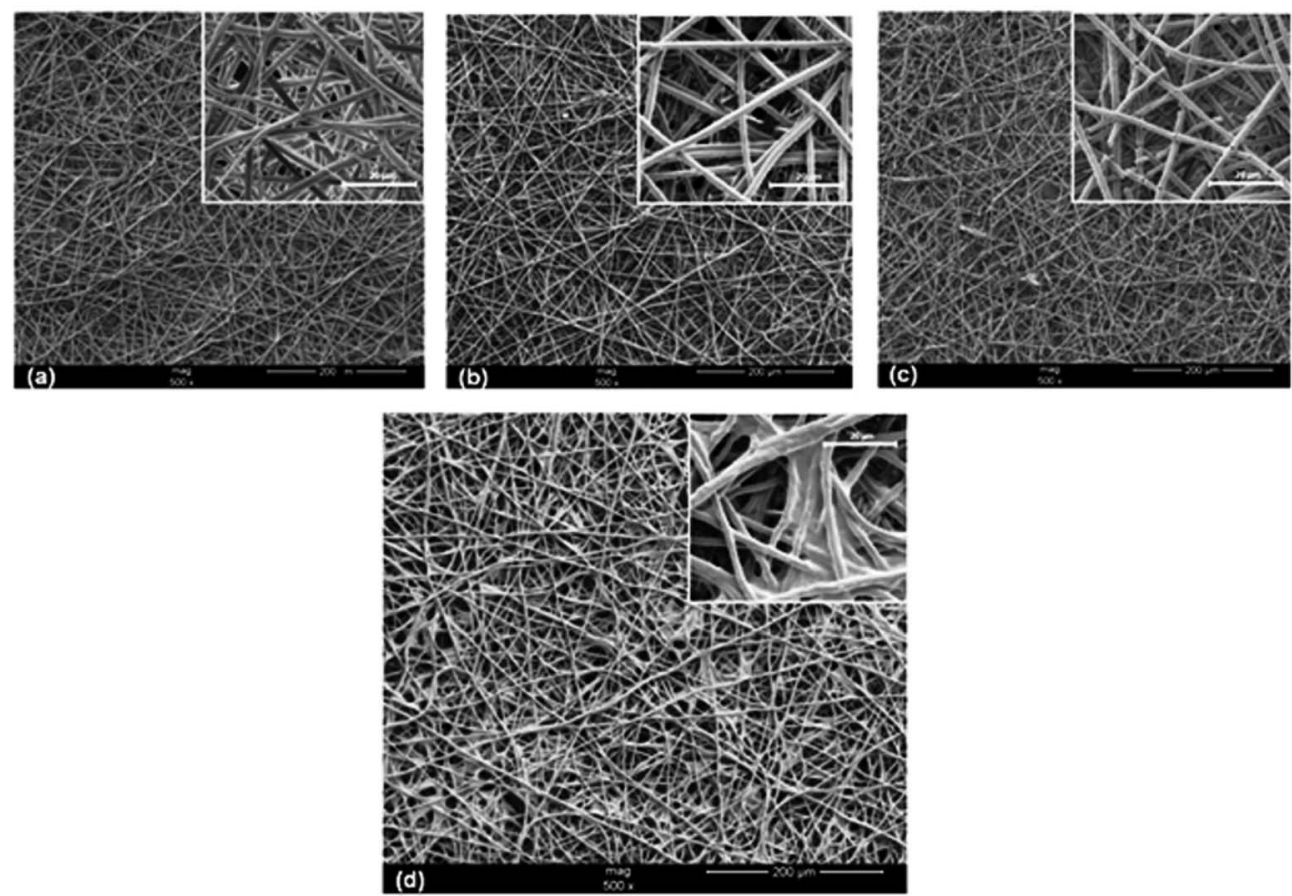

Fig. 5 SEM images of untreated PCL NFMs (a), UV/O 3 -treated PCL NFMs during 1 min (b) and 5 min (c), and CS-immobilized PCL NFMs (d). [Source: Piai et al., ${ }^{41}$ Copyright@ 2017 with permission from Elsevier B.V.] 

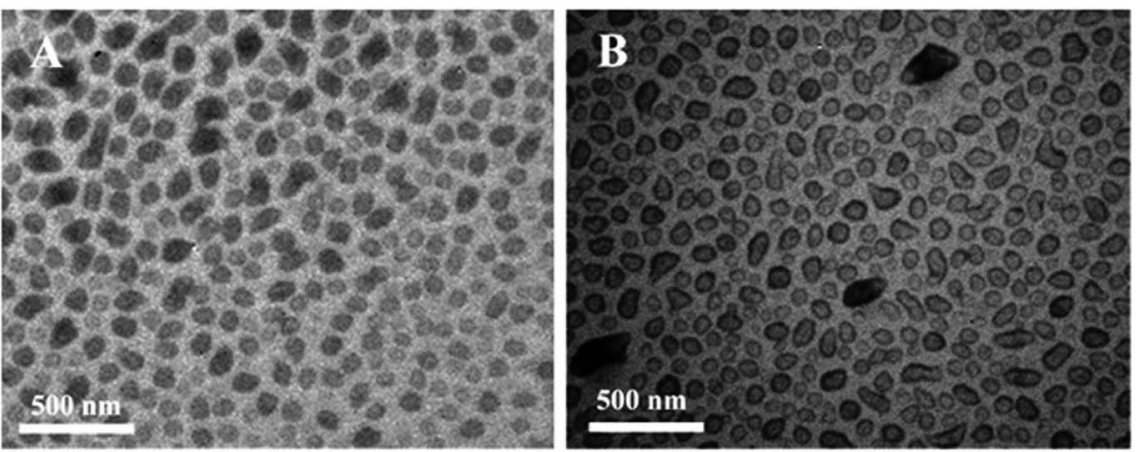

Fig. 6 TEM images of selenium-chondroitin sulfate (SeCS) nanoparticles dispersed in distilled water (A) and ethanol (B). [Source: Han et al., ${ }^{43}$ Copyright@ 2012 with permission from Elsevier B.V.]

\subsection{Fucosylated chondroitin sulfate from Pearsonothuria graeffei by human gut microflora}

A highly repeated structure from Pearsonothuria graeffei was used as a source to isolate a highly repeated structure of fucosylated chondroitin sulfate (FCS-pg); it was subjected to an in vitro fermentation model to investigate its fermentability and was also studied for its effects on human gut microflora. The fermentation of FCS-pg into short chain fatty acids (SCFAs) by gut microflora was analyzed by high performance liquid chromatography (HPLC) measurements of partial human fecal samples. 16S rRNA gene-based polymerase chain reaction-based denaturing gradient gel electrophoresis (PCR-DGGE) profiling and real-time quantitative PCR analysis confirmed increased proportions of Clostridium cluster XI, Bacteroides prevotella group, Bifidobacterium genus, Clostridium cluster I and Clostridium cluster XIVab in FCS-pg but decreased concentrations of Enterobacteriaceae and Lactobacillus. Bacteroides, Bifidobacterium and Clostridium mainly helped to ferment FCS-pg, which increased the content of probiotic bacteria; this is slightly different from most sulfated polysaccharides procured from marine animals. The study also provided useful information about the mechanism of absorption and the functional activity of FCS-pg within the gastrointestinal tract of the human body. ${ }^{45}$

\subsection{Novel biological roles of fucosylated chondroitin sulfates isolated from the sea cucumber Apostichopus japonicus exhibit neurite outgrowth promoting activity}

Chondroitin sulfate (CS) contains sulfated glycosaminoglycan (GAG) chains that consist of repeating disaccharide units of glucuronic acid (GlcA) and $N$-acetylgalactosamine (GalNAc). A fucosylated chondroitin sulphate (FCS) was isolated from the body wall of the sea cucumber Apostichopus japonicus and had promotional effectivity on neurite outgrowth; meanwhile, FCS trisaccharide $\beta$-D-GalNAc (4,6-O-disulfate) $(1-4)[\alpha$-L-fucose $(2,4-$ $O$-disulfate) (1-3)]- $\beta$-D-GlcA was chemically synthesized. The FCS polysaccharides were composed of the E-type disaccharide unit GlcA-GalNAc (4,6-O-disulfate) with distinct sulfated fucose branches. Although their structures were composed of lower $\mathrm{E}$ units, the FCS polysaccharides showed more effective neurite outgrowth-promoting activity than squid cartilage-derived CS-E polysaccharides, which are characterized by their predominant $\mathrm{E}$ units; this suggested potential roles of the fucose branch in neurite outgrowth. Indeed, the chemically synthesized FCS trisaccharide was as effective as CS-E tetrasaccharides in stimulating neurite elongation in vitro. ${ }^{46}$

\subsection{Chondroitin sulphate isolated from chicken keel bone for potential biomedical applications}

A newer chondroitin sulfate (CS) was isolated from chicken keel bone cartilage for future use in tissue engineering and the pharmaceutical industry. High pressure size exclusion chromatography analysis data showed two peaks at $100 \mathrm{kDa}$ for CSKeel polysaccharide and $1 \mathrm{kDa}$ protein. The polydispersity of CS-Keel was revealed by DLS analysis. CS-Keel yielded $15 \%$ and $53 \pm 5 \%$ uronic acid content. In CS-Keel, $58 \%$ and $42 \%$ of UAGalNAc4S and UA-GalNAc6S disaccharide were present, respectively. FT-IR spectroscopy confirmed that CS-Keel is chondroitin 4-sulphate, and ${ }^{1} \mathrm{H}$ NMR spectroscopy confirmed the presence of $N$-acetylgalactosamine and glucuronic acid in CS-Keel. The layer structure and size of the CS-Keel fibres were confirmed by FESEM and AFM data. DSC, TGA and DTG studies revealed that the $T_{\mathrm{d}}$ (thermal degradation temperature) of CSKeel was $243{ }^{\circ} \mathrm{C}$. The biocompatibility of CS-Keel was confirmed by in vitro cell proliferation assays and morphological analysis of mouse fibroblast L929 cell lines. Approximately $49 \%$ antioxidant activity was shown by CS-Keel $\left(5 \mathrm{mg} \mathrm{mL}{ }^{-1}\right)$ using DPPH, as well as $22 \%$ protection from oxidative damage by superoxide radical. CS-Keel was confirmed to have better emulsifying activity $(70.3 \%)$ than commercial sodium alginate $(60.2 \%){ }^{47}$

\subsection{Fucosylated chondroitin sulfate oligosaccharides exert anticoagulant activity}

Two sea cucumbers, I. badionotus (fCS-Ib) and P. graeffei (fCS$\mathrm{Pg}$ ), were used to prepare a series of pure fucosylated CS oligosaccharides using a controllable fenton depolymerization reaction. NMR and ES-MS analysis of the fucosylated CS oligosaccharide fractions with different sulfation patterns indicated that unlike other chemical methods and acid hydrolysis, freeradical depolymerization was highly selective and 
preferentially cleaved the GlcA in the backbone without influencing Fuc branching or sulfation. For the native fCS and fCS oligosaccharides, the fCS oligosaccharides showed better anticoagulant activity only through the intrinsic pathway and had no or little effect on the extrinsic coagulation pathways. The molecular weight of fCS was proportional to its APTT activity. Moreover, anticoagulant factor assays revealed that the two fCSs sharply decreased thrombin-mediated inhibition through ATIII/HCII upon depolymerization, and fCS-4-fCS-D6 showed stronger anti-Xase activity than anti-FXa activity and anti-FIIa activity mediated by AT and HCII. These outcomes suggested that native fCSs acts on both serpin-related and serpinindependent pathways. fCS oligosaccharides exerted activity on coagulation through serpin-independent pathways while targeting the intrinsic tenase complex rather than anticoagulant serpin-related systems. Therefore, the sulfation pattern of the fucose branch plays an important role in the anticoagulant activity. ${ }^{48}$

\subsection{Chondroitin sulfate-functionalized polyamidoamine as a tumor-targeted carrier for miR-34a delivery}

The Michael addition reaction was used to develop a chondroitin sulfate (CS) polyamidoamine dendrimer (CS-PAMAM) as a tumor-targeted carrier for miR-34a delivery. Efficient cellular uptake of an miR-34a derivative of CS-PAMAM was achieved in CD44-dependent endocytosis, which facilitated the endosomal escape of miR-34a after $4 \mathrm{~h}$. Cell apoptosis and cell cycle arrest were enhanced through inhibition of cell proliferation by miR-34a delivery; inhibition of cell migration and cell invasion was also observed. Human lung adenocarcinoma A549 cells were inhibited by intravenous injection of the CS-PAMAM/ miR-34a formulation, which induced tumor apoptosis with enhanced accumulation of miR-34a in tumor tissue. Moreover, CS-PAMAM is a potential tumor-targeted oligonucleotide carrier for proper tumor gene therapy. ${ }^{49}$

\subsection{The roles of chondroitin sulfate and sulfated} hyaluronan in skin tissue engineering

Electrospun nanofibrous scaffolds were developed using gelatin and different concentrations of chemically sulfated or nonsulfated hyaluronan (sHA or HA) and chondroitin sulfate (CS). Fiber morphology was observed, with no differences between varying concentrations and types of glycosaminoglycans (GAGs). In vitro release kinetics data confirmed that the release of GAGs was driven by a diffusion mechanism. Adhesion and proliferation studies of human keratinocyte (HaCaT), fibroblast (Hs27) and mesenchymal stem cells (hMSCs) were used to analyze the effects of the scaffolds. The cell numbers increased by 5 times when cultivating all three cell types alone on scaffolds containing HA and CS. These outcomes revealed that sulfated GAG-containing electrospun nanofibrous scaffolds are beneficial for the development of effective skin tissue engineered constructs by stimulating cellular performance and therefore accelerating epidermal-dermal regeneration processes. $^{50}$

\subsection{Chondroitin sulfate glycosaminoglycan in NEDD9- mediated breast cancer cell growth}

NEDD9 (Cas-L, HEF-1) plays an important role in the development, progression, and metastasis of breast cancer cells. NEDD9 plays a critical role in promoting the migration and growth of MDA-MB-231. Stable cells overexpressing NEDD9 were developed to characterize the mechanisms of NEDD9mediated cancer migration and growth using HCC38, which expresses a low level of endogenous NEDD9, as a parental cell line. Microarray studies revealed that the core proteins CD44 and Ser-glycin were markedly upregulated in HCC38 (NEDD9) cells compared to HCC38 (Vector) cells, whereas syndecan-1, syndecan-2, and versican were downregulated in HCC38 (NEDD9) cells. Enzyme-generated chondroitin sulfate glycosaminoglycans (CS) such as CHST11, CHST15, and CSGALNACT1 were upregulated in HCC38 (NEDD9) cells compared to HCC38 (Vector) cells. Immunofluorescence studies using the specific antibody GD3G7 confirmed the overexpression of the CS-E subunit in HCC38 (NEDD9) cells. Immunoprecipitation and western blotting analysis confirmed that CS-E was attached to the CD44 core protein. Removal of CS by chondroitinase ABC significantly inhibited the anchorage-independent colony formation of HCC38 (NEDD9) cells in methylcellulose. GD3G7 significantly inhibited the colony formation of HCC38 (NEDD9) cells, which suggested that the CS-E subunit plays an important role in this process. Moreover, treatment of HCC38 (NEDD9) cells with chondroitinase ABC or GD3G7 significantly inhibited mammosphere formation. Exogenous addition of CS-E increased the colony formation and mammosphere formation of HCC38 parental and HCC38 (Vector) cells. These outcomes suggested that NEDD9 regulates the synthesis and expression of tumor-associated glycocalyx structures. ${ }^{51}$

\subsection{Highly sulfated chondroitin sulfates in ovarian cancer defined by the single chain antibody GD3A11}

Chondroitin sulfate (CS), a class of sulfated polysaccharides, is abundantly present in the extracellular matrix (ECM) of ovarian cancer cells. Structural alterations of CS chains play a role in cancer development and progression. Highly sulfated CS was used as a biomarker in ovarian cancer using the single chain antibody GD3A11, which was selected by phage display technology. Indirect ELISA was used to determine the specificity of the antibody. Immune histochemistry was used to assess GD3A11 epitope expression in healthy organs and benign and malignant ovarian tumors $(N=359)$, and the outcomes were correlated with clinical parameters. The CHST15 gene is responsible for the biosynthesis of highly sulfated CS and has been studied for mutation and methylation status. In normal organs, the GD3A11 epitope was minimally expressed. More intense expression of ECM was observed in the different ovarian cancer subtypes than in benign ovarian tumors. Tumor grade, FIGO stage, and the use of chemotherapy did not affect the expression of ECM. Intense expression was identified for the aggressive ovarian cancer phenotype as an independent predictor for poor prognosis. CHST15 gene analysis showed neither mutations nor an altered methylation status. Specific 
highly sulfated CS motifs were expressed in the tumoral ECM; they are potential biomarkers in ovarian cancer patients. These matrix motifs constitute a novel class of biomarkers with prognostic significance and are instrumental for innovative diagnostic and therapeutic applications in the management of ovarian cancer. ${ }^{52}$

\subsection{Chondroitin sulfate-cysteine conjugates as intra- articular agents}

A chondroitin sulfate-cysteine conjugate (CS-cys) was used as a novel bioadhesive agent for intraarticular use. A polymermucus mixture and the rotating cylinder method were used to investigate the mucoadhesive properties of the synthesized CScys, while the bioadhesive features of CS-cys on porcine articular cartilage were evaluated via tensile studies. Thiolation was achieved by attachment of L-cysteine to CS via amide bond formation mediated by carbodiimide as a coupling reagent. The conjugate exhibited $421.17 \pm 35.14 \mu \mathrm{mol}$ free thiol groups per gram of polymer. The reduced CS-cys displayed 675.09 $\pm 39.67 \mu \mathrm{mol}$ free thiol groups per gram of polymer after disulfide bond reduction using tris(2-carboxyethyl)phosphine hydrochloride. An increase in the dynamic viscosity of thiolated CS due to oxidative disulfide bond formation was demonstrated using a capillary viscometer. The combination of CS-cys and mucus led to a 4.57-fold increase in dynamic viscosity in comparison with a mucus control. Furthermore, the adhesion time to porcine mucosa of a CS-cysbased test disk was enhanced by 2.48 -fold compared to unmodified CS as measured by the rotating cylinder method; this suggests interactions between thiomers and the mucus gel layer via disulfide bond formation. Tensile studies of thiolated CS on porcine articular cartilage showed 5.37 and 1.76-fold increases in the total work of adhesion and the maximum detachment force, respectively, in comparison with unmodified CS; this indicates the bioadhesive features of CS-cys. The cytotoxicity of CS-cys was assessed in Caco- 2 cells and rat primary articular chondrocytes using MTT and LDH release assays; the results demonstrated the safety of CS-cys at a concentration of $0.25 \%(\mathrm{w} / \mathrm{v})$ in Caco- 2 cells. Furthermore, $0.1 \%$ CS-cys was found to be non-toxic to rat primary articular chondrocytes. According to these results, CScys possesses improved bioadhesive properties and may be useful as an intra-articular agent for treatment of osteoarthritis. ${ }^{53}$

\subsection{Novel chondroitin lyase ODV-E66, a baculovirus envelope protein}

Chondroitin lyases are known to be pathogenic bacterial enzymes that degrade chondroitin. Recently, baculovirus envelope protein ODV-E66 was identified as the first reported viral chondroitin lyase. ODV-E66 has low sequence identity with bacterial lyases at $<12 \%$ and unique characteristics reflecting the life cycle of baculovirus. To understand the structural basis of ODV-E66, its crystal structure was determined; it was found that its structural fold resembled that of polysaccharide lyase 8 proteins and that the catalytic residues were also conserved. This structure enabled discussion of the unique substrate specificity and stability of ODVE66 as well as the host specificity of baculoviruses. ${ }^{54}$

\subsection{Polyelectrolyte complexes of poly[(2-dimethylamino)} ethyl methacrylate]/chondroitin sulfate: preparation, characterization, cytotoxicity and controlled release of chondroitin sulfate

A novel polyelectrolyte complex was prepared using poly[(2dimethylamino) ethyl ethacrylate] (PDMAEMA) and chondroitin sulfate (CS), and its properties were investigated by wide angle X-ray scatttering, FTIR, TGA, SEM and DLS analysis. At $\mathrm{pH}$ 6.0, 7.0 and 8.0, PDMAEMA/CS PECs showed hydrophilichydrophobic transitions, whereas at $\mathrm{pH}$ 8.0, non-complexed PDMAEMA showed a transition. The complexation between CS and PDMAEMA increased the biocompatibility of the PECs as per cytotoxicity assays using healthy VERO cells related to PDMAEMA, and the biocompatible behavior was dependent on the amount of CS present in the PECs. ${ }^{55}$

\subsection{Chondroitin sulfate modulates inflammation and atherogenesis in obesity}

Chondroitin sulfate (CS) treatment affected the formation of atheroma via interactions with endothelial cells and monocytes. Arterial atheromatous plaques were characterized by multiphoton microscopy and serum proinflammatory cytokines by immuneenzymatic techniques in obese mice with administration of CS ( $1 \mathrm{~g}$ per $\mathrm{kg}$ per day, i.p.) for 6 days. Western blots, immunoenzymatic techniques and transwell migration assays helped evaluate the effects of CS on signaling pathways, cytokine secretion and macrophage migration in cultures of human coronary endothelial cells and in a monocyte cell line stimulated with TNF- $\alpha$. Decreased extension of foam cell coverage in atheromatous plaques of arterial bifurcations by $62.5 \%$ was observed; the serum concentration of IL1- $\beta$ decreased by $70 \%$, TNF- $\alpha$ decreased by $82 \%$ and selected chemokines decreased by $25 \%$ to $35 \%$. Coronary endothelial cells and monocyte cultures with increased TNF- $\alpha$ secreted fewer proinflammatory cytokines in the presence of CS; it also decreased the activation of the TNF- $\alpha$ signaling pathway in endothelial cells, decreased the propagation of inflammation and prevented the formation of atherosclerotic plaques. ${ }^{56}$

\subsection{In situ synthesis of nano-hydroxyapatite/chitosan/ chondroitin sulfate/hyaluronic acid for bone tissue engineering}

Nano hydroxyapatite (nHAP), chitosan (CS), chondroitin sulfate (CSA) and hyaluronic acid (HA) were used to develop a biomimetic calcium phosphate mineralized organic-inorganic micro/ nanostructure hybrid scaffold by freeze-drying technology. Fourier transform infrared spectroscopy (FTIR) and X-ray diffraction (XRD) were used to characterize the chemical structure, and the surface morphology was characterized by FTIR, XRD and scanning electron microscopy (SEM); the scaffold was evaluated to have well-distributed pore sizes and proper mechanical strength due to the addition of nHAP and the interactions between the positively charged CS and the negatively charged CSA and HA. The biocompatibility factor of the scaffold was characterized by MTT cytotoxicity assay, alkaline phosphatase (ALP) activity and Hoechst 33258 fluorescence 
staining methods. All outcomes indicated that the scaffolds possessed good biocompatibility and enhanced proliferation and differentiation of osteoblasts. Therefore, in situ biomimetic mineralized nHAP/CS/CAS/HA hybrid scaffolds are promising candidates for bone tissue engineering. ${ }^{57}$

\subsection{Inhibition of cell proliferation and migration by chondroitin sulfate- $g$-polyethylenimine-mediated miR-34a delivery}

Chemically conjugated chondroitin sulfate and PEI25K through Michael addition was used to construct a non-viral gene carrier, CS-PEI, for the delivery of miR-34a to achieve inhibition of cell proliferation and migration using PC-3 prostate tumor cells as a model. The CS-PEI and miR-34a nanoparticles were prepared and characterized with a particle size and zeta potential of $170.7 \mathrm{~nm}$ and $+42.2 \mathrm{mV}$, respectively. Flow cytometry and fluorescence microscopy revealed that CS-PEI efficiently induced cellular uptake of miR-34a by CD44-mediated endocytosis. The percentage of early apoptotic cells was $47.49 \%$; meanwhile, the activation of caspase-3, -8 and -9 and decreased expression levels of Bcl-2 were observed by CS-PEI-mediated miR-34a transfection, along with obvious cell apoptosis. CS-PEI/miR34a transfection inhibited cell migration, which was observed by a wound healing assay. Therefore, CS-PEI can potentially be employed as a promising tumor-targeting system for miR-34a delivery in tumor gene therapy. ${ }^{58}$

\subsection{Hydrogels of hyaluronic acid and chondroitin sulfate as potential cell scaffold materials}

A new cell scaffold material was developed for cellular adhesion, proliferation and differentiation using natural polysaccharides due to their excellent biocompatibility, biodegradability, and biofunctions. However, the application of natural polysaccharides is often severely limited by their mechanical behavior. This study revealed that a tough and elastic hydrogel scaffold could be developed with hyaluronic acid (HA) and chondroitin sulfate (CS). Hyaluronic acid, chondroitin sulfate and tyramine (TA) were amalgamated, and ${ }^{1} \mathrm{H}$ NMR studies demonstrated that the degrees of substitution (DS) were $10.7 \%$ and $11.3 \%$, respectively. In the presence of $\mathrm{H}_{2} \mathrm{O}_{2}$ and horseradish peroxidase enzyme, HA-TA and CS-TA hydrogels were prepared. SEM, static and dynamic mechanical properties were determined by a Shimadzu electromechanical testing machine and Q800 dynamic mechanical thermal analyzer to characterize the sectional morphologies of the hydrogels. All hydrogels were observed to have good ability to recover their initial appearance after deformation, with high storage moduli $\left(E^{\prime}\right)$ of the hydrogels, and they also demonstrated fatigue resistance to cyclic compression. Significant cell viability as detected by confocal laser scanning microscopy was observed in the case of mesenchymal stem cells encapsulated in hydrogels. These outcomes revealed that the hydrogels possessed both good mechanical properties and biocompatibility and may serve as model systems to explore mechanisms of deformation and energy dissipation. ${ }^{59}$

\subsection{Anticoagulant and antithrombotic evaluation of native fucosylated chondroitin sulfates}

A structurally unusual glycosaminoglycan, fucosylated chondroitin sulfate, was observed to have distinct anticoagulant properties; especially, it was a strong inhibitor of the intrinsic factor Xase (anti-Xase). This study involved the purification of six native fucosylated chondroitin sulfates with variable sulfation patterns a series of FCS derivatives were prepared as highly selective inhibitors of human Xase. Three fucosylated chondroitin sulfates containing higher Fuc2S4S exhibited stronger AT-dependent anti-IIa activity; meanwhile, the other molecules containing more Fuc3S4S produced potent HCII-dependent anti-IIa activities. A minimum of 6 to 8 trisaccharide units with free carboxyl groups and full fucosylation of GlcA were required for potent anti-Xase activity, and approximately six trisaccharide units and partial fucosylation of GlcA contributed to potent HCII-dependent activity. AT-dependent anti-IIa activities decreased with decreasing molecular weight, along with elimination of human platelets and factor XII activation. In vitro and in vivo studies revealed that compounds with 6 to $12 \mathrm{kDa}$ fractions could be used as putative selective intrinsic Xase inhibitors with antithrombotic action. ${ }^{60}$

\subsection{Chondroitin sulfate and kallikrein in saliva: markers for glossodynia}

Glossodynia or burning mouth syndrome is a multifunctional disorder where the oral mucosa of patients are apparently normal but they experience burning, dry mouth, and painful tongue and lips. In glossodynia, patients were reported to have different biochemical and physiological markers in saliva compared to normal subjects. Saliva-buffering capacity and the presence of protein and hyaluronic acid were observed here. Also, chondroitin sulfate (CS) concentration was decreased in the saliva of patients with glossodynia compared to a control group ( $p=0.0036$ ), and the content of glandular kallikrein was observed to be increased in the saliva of glossodynia patients compared to normal subjects $(p<0.0001)$. These results indicated involvement of the kinin system, possibly related to low levels of CS. Furthermore, the level of serotonin was found to be depressed in the serum of glossodynia patients $(p=0.0478) .{ }^{61}$

\subsection{Chondroitin sulfate-mediated $\mathbf{N}$-cadherin/ $\beta$-catenin signaling associated with basal-like breast cancer cell invasion}

Metastasis of tumors involves cancer cell invasion within membranes and interstitial tissues. The first invasion step is adherence of tumor cells to the extracellular matrix (ECM); this binding navigates various signals from the ECM to tumor cells, and it is challenging to establish the mechanisms behind this regulation of tumor cell invasion. This study observed that the extracellular component chondroitin sulfate was responsible for the invasion of basal-like breast cancer cell line BT-549. Interaction and induction of chondroitin sulphate increased due to the proteolytic cleavage of N-cadherin in BT-549 cells, and it was observed that a C-terminal intracellular $\mathrm{N}$-cadherin fragment formed a complex with $\beta$-catenin. Also, this cleavage 
of $\mathrm{N}$-cadherin increased cytoplasmic and nuclear $\beta$-catenin levels and increased the matrix metalloproteinase 9 (MMP9) gene (target of $\beta$-catenin nuclear signaling). Furthermore, it was observed that $\mathrm{CS}$-induced $\mathrm{N}$-cadherin proteolysis required a caveolae-mediated endocytosis process, while nystatin blocked both chondroitin sulphate-induced endocytosis and proteolytic cleavage of $\mathrm{N}$-cadherin and inhibited BT-549 cell invasion. Therefore, with the inhibition of chondroitin 4-Osulfotransferase-1 biosynthetic enzyme, activation of the $\mathrm{N}$ cadherin/ $\beta$-catenin pathway was suppressed by $\mathrm{N}$-cadherin endocytosis and a statistical decrement in BT-549 cell invasion was observed. These results revealed that chondroitin sulfates produced by C4ST-1 can be used as therapeutic targets for the treatment of basal-like breast cancers. ${ }^{62}$

\subsection{Development of affinity-based delivery of NGF from a chondroitin sulfate biomaterial}

This study reflected the effects of affinity-based delivery of C6S and NGF from gels on cortical neurite behavior by incorporating C6S and NGF into PEG gels through C6S binding peptides. Gels embedded with C6S inhibited neurite outgrowth, whereas gels with NGF promoted neurite extension. NGF was used to overcome the inhibitory activity of $\mathrm{C6S}$, which was slowly released from the PEG gels through non-covalent interactions between C6S binding peptide, C6S and NGF. The affinity-based system was developed with C6S and NGF, which are considered to be ideal biomaterials for neural development and for neural tissue engineering. ${ }^{63}$

\subsection{CS formulation in the treatment of psoriasis}

The main interest behind this research was to treat psoriasis with invasive infection. To achieve this, sodium CS (molecular weight: 10000 to 20000 daltons) with $5 \%$ to $7 \%$ sulfur content was applied. The preferred content of sodium CS on a daily basis was within 200 to $3000 \mathrm{mg}$, which was administered by oral formulation. Finally, after two months of treatment with sodium CS (800 mg per day), lower numbers of plaque forming cells (decreased by $28 \%$ ) and denser epidermal cells $(400 \mu \mathrm{M})$ with greater thickness in the corneal region $(160 \mu \mathrm{M})$ and basal corneal region $(344 \mu \mathrm{M})$ were observed. This information was directly correlated with the effectivity of CS on psoriasis. ${ }^{64}$

\subsection{A CS formulation as an intra-articular gel for treatment of articular damage}

The main focus behind this invention was bypassing the parenteral route of chondroitin proteoglycans and developing an intra-articular gel that could be directly applied on the site. The principal problem associated with this formulation was the generation of an immunological reaction after four hours at the site of application. In this way, numerous hit and trial experiments were performed with amounts of protein in CS as low as $0.2 \mathrm{mg} \mathrm{g}^{-1}$ of CS; based on the results, a minimum of one local anesthetic (prilocaine) was chosen. Also, the amount of protein was mainly preferred to be within the range of 0.15 to $0.05 \mathrm{mg}$ per gram of CS. Along with the main constituents, sodium dihydrogen orthophosphate and disodium hydrogen orthophosphate were the main fillers in the formulation. This formulation was highly promising to treat damage of cartilages and tendons. ${ }^{65}$

\subsection{Fibrillogenetic applications of CS-E}

In this work, the agonstic and antagonistic effects of CS-E were the initial focus. CS-E agonistically facilitated the internal wound healing process, and the antagonistic pathway of CS-E involved the inhibition of fibril formation in myocardial infraction. However, in the positive direction, CS-E affected internal wound healing processes, as determined by in vitro and in vivo fibril formation studies. The results revealed that a CS-E concentration of 0.13 to $30 \mu \mathrm{g} \mathrm{mL}^{-1}$ demonstrated greater applicability as a fibrillogenetic agent. ${ }^{66}$

\section{Conclusion}

This article revealed that chondroitin sulphate can be extracted from $S$. maximus, $H$. scabra, E. fraudatrix, M. magnum, and $H$. mexicana and is bestowed with anticoagulant, articular cartilage repair, corneal lesion healing, antidiabetic, antiproliferative, anti-HSV2, anti-angiogenic, and antibacterial effects. It is also effective in the treatment of osteoarthritis and Kashin-Beck disease (KBD). Therefore, chondroitin is a biomarker that may have immense biomedical applications, as described above. This review especially emphasized various biomedical applications of chondroitin (sulfate) in the field of biomedical research in the present as well as in the future.

\section{Funding information}

Dr Dilipkumar Pal (Author) and Dr Supriyo Saha (Co-author) did not receive any fund, projects or support from any funding agency to bear the expenses of APC/Publication charges. They are really thankful to Royal Society of Chemistry for waiving the APC/Publication charges.

\section{Conflicts of interest}

There is no conflict of interest to declare.

\section{References}

1 D. Pal, A. Nayak and S. Saha, Natural polymers for Pharmaceutical Applications, Apple Academic Press, USA, 2019, vol. 3, in press.

2 D. Pal, T. De and A. Baral, PHARMANEST, 2013, 4, 54-65.

3 S. B. Nimse and D. Pal, RSC Adv., 2015, 5, 27986-28006.

4 D. Pal, S. Saha, A. Nayak and M. S. Hansain, Natural polymers for Pharmaceutical Applications, Apple Academic Press, USA, 2019, vol. 2, in press.

5 A. Nayak and D. Pal, Encyclopedia of Biomedical Polymers and Polymer Biomaterials, Taylor \& Francis, New York, 1st edn, 2015, vol. 8.

6 D. Pal and A. Nayak, Encyclopedia of Biomedical Polymers and Polymer Biomaterials, Taylor \& Francis, New York, 1st edn, 2015, vol. 6 . 
7 A. Nayak, D. Pal and K. Santra, Int. J. Biol. Macromol., 2014, 66, 203-211.

8 D. Pal and A. Nayak, Drug Delivery, 2012, 125-131.

9 A. Nayak and D. Pal, Carbohydr. Polym., 2014, 103, 154-163.

10 J. K. Alma, H. M. E. Gerard, K. L. M. Tom, S. C. S. Stefaan, D. Joseph, K. Jeroen, A. J. Z. Sebastian, D. Jacob and F. Jan, Macromol, 2000, 33, 3705-3713.

11 R. Bagari, D. Bansal, A. Gulbake, A. Jain, V. Soni and S. K. Jain, J. Drug Targeting, 2011, 19(4), 251-257.

12 S. M. Dyck and S. K. Abdolrezaee, Exp. Neurol., 2015, 269, 169-187.

13 P. R. Guru, H. Bera, M. Das, M. S. Hasnain and A. K. Nayak, Starch/Staerke, 2018, 70, 1700136.

14 M. S. Hasnain, P. Rishishwar, S. Rishishwar, S. Ali and A. K. Nayak, Int. J. Biol. Macromol., 2018, 116, 1074-1081.

15 L. Hongxia, W. Shuqin, Y. Jingmou, F. Dun, R. Jin, L. Zhang and J. Zhao, Mater. Sci. Eng., C, 2017, 75, 55-63.

16 X. F. Yin, L. L. Wang and X. C. Chu, Mater. Sci. Eng., C, 2014, 78, 452-456.

17 F. B. Ma, N. Liu, N. Hu, C. Y. Wen and B. Tang, Carbohydr. Polym., 2017, 170, 217-225.

18 R. Zhenkun, J. Yuanyuan, Y. Wang and L. Dong, Int. J. Biol. Macromol., 2018, 108, 1158-1164.

19 L. Yang, Y. Wang, S. Yang and Z. Lv, Int. J. Biol. Macromol., 2018, 108, 710-718.

20 N. E. Ustyuzhanina, M. I. Bilan, A. S. Dmitrenok, N. E. Nifantiev and A. I. Usov, Carbohydr. Polym., 2017, 164, 8-12.

21 F. Zhou, X. Zhang, D. Cai, J. Li, Q. Mu, W. Zhang, S. Zhu, Y. Jiang, W. Shen, S. Zhang and H. W. Ouyang, Acta Biomater., 2017, 63, 64-75.

22 N. E. Ustyuzhanina, M. I. Bilan, A. S. Dmitrenok, E. Y. Borodina, V. A. Stonik, N. E. Nifantiev and A. I. Usov, Carbohydr. Polym., 2017, 167, 20-26.

23 M. Jiaojiao, C. Wang, L. Wenjing and J. Yang, Int. J. Biol. Macromol., 2017, 98, 208-215.

24 S. Giuseppina, M. C. Bonferoni, S. Rossi, A. Delfino, F. Riva, A. I. Cornaglia, G. Marrubini, G. Musitelli, C. D. Fante, C. Perotti, C. Caramella and F. Ferrari, Int. J. Pharm., 2016, 509, 188-196.

25 Y. Hattori, A. Nakamura, S. Hanaya, Y. Miyanabe, Y. Yoshiike, T. Kikuchi, K. Ozaki and H. Onishi, J. Drug Delivery Sci. Technol., 2017, 41, 401-409.

26 L. Qinying, C. Chao, C. Yaoguang, F. Z. Zhang, J. L. Robert, X. Changhu, L. Guoyun and Y. Guangli, Carbohydr. Polym., 2018, 181, 1160-1168.

27 F. Krichen, N. Volpi, A. Sila, F. Maccari, V. Mantovani, F. Galeotti, S. E. Chaabouni and A. Bougatef, Int. J. Biol. Macromol., 2017, 95, 32-39.

28 S. M. Ibrahim, I. Althagafi, H. D. Takagi and R. M. Hassan, J. Mol. Liq., 2017, 244, 353-359.

29 L. Tio, C. Orellana, S. Perez-Garcia, L. Piqueras, P. Escudero, Y. Juarranz, N. Garcia-Giralt, M. Francisco, A. Farrana, P. Benitoa, R. P. Gomarizc, S. Maria-Jesus and J. Monforta, Med. Clin., 2017, 149(1), 9-16.
30 M. Moto, N. Takamizawa, T. Shibuya, A. Nakamura, K. Katsuraya, K. Iwasaki, K. Tanaka and A. Murota, J. Funct. Foods, 2018, 40, 336-340.

31 K. Ikuko, K. Hideyo, C. Fengchao and E. Masahiko, Carbohydr. Polym., 2015, 121, 362-371.

32 H. Yuan, J. Xue, B. Qian, H. Chen, Y. Zhu and M. Lan, Appl. Surf. Sci., 2017, 394, 403-413.

33 A. Galus, J. M. Mallet, D. Lembo, V. Cagno, M. Djabourov, H. Lortat-Jacob and K. Bouchemal, Carbohydr. Polym., 2016, 136, 113-120.

34 S. Qingsen, S. Jingjing, S. Guanrui, Z. Meifang, C. Chao, H. Jiejie, L. Guoyun and Y. Guangli, Int. J. Biol. Macromol., 2016, 89, 489-498.

35 K. Takashi, K. Ikuko, N. Hiroyuki and N. Toshiya, Biochem Biophys Rep, 2017, 9, 72-78.

36 N. Wataru, Y. Kotaro, O. Yoshiki, N. Osamu, O. Katsuhito, S. Hidetomo and K. Tan-No, J. Pharmacol. Sci., 2016, 131, 275-278.

37 L. Xiangjun, H. Kurita, T. Xiao, K. Lijima, K. Kenji and N. Jun, Acta Histochem., 2017, 119(5), 439-445.

38 H. Yuan, B. Qian, H. Chen and M. Lan, Appl. Surf. Sci., 2017, 426, 587-596.

39 L. Xiaoxiao, H. Jiejie, S. Xindi, Z. Xiao, Z. Xiaoliang, L. Qinying, W. Xiaojiang, C. Chao, L. Guoyun and Y. Guangli, Carbohydr. Polym., 2016, 152, 343-350.

40 J. Han, D. Li, C. Qu, D. Wang, L. Wang, X. Guo and M. J. Lammi, Osteoarthr. Cartil., 2017, 25(8), 1372-1375.

41 J. F. Piai, M. Alves da Silva, A. Martinsa, A. B. Torress, S. Faria, R. L. Reis, E. C. Muniz and N. M. Neves, Appl. Surf. Sci., 2017, 403, 112-125.

42 N. E. Ustyuzhanina, M. I. Bilan, A. S. Dmitrenok, E. A. Tsvetkova, A. S. Shashkov, V. A. Stonik, N. E. Nifantiev and A. I. Usov, Carbohydr. Polym., 2016, 153, 399-405.

43 J. Han, X. Guo, Y. Lei, B. S. Dennis, S. Wu and C. Wu, Carbohydr. Polym., 2012, 90, 122-126.

44 J. R. Dorvee, L. Gerkowicz, S. Bahmanyar, A. Deymier-Black and A. Veis, Arch. Oral Biol., 2016, 62, 93-100.

45 C. Y. Wei, N. B. Liao, Y. Zhang, X. Q. Ye, S. Li, Y. Q. Hu, D. H. Liu, R. J. Linhardt, X. Wang and S. G. Chen, Int. J. Biol. Macromol., 2017, 102, 1195-1201.

46 M. Shida, T. Mikami, J. Tamura and H. Kitagawa, Biochem. Biophys. Res. Commun., 2017, 487, 678-683.

47 A. Rani, R. Baruah and A. Goyal, Carbohydr. Polym., 2017, 159, 11-19.

48 J. Li, S. Li, L. Yan, T. Ding, R. J. Linhardt, Y. Yu, X. Liu, D. Liu, X. Ye and S. Chen, Euro J Med Chem, 2017, 139, 191-200.

49 W. Chen, Y. Liu, X. Liang, Y. Huang and Q. Li, Acta Biomater., 2017, 57, 238-250.

50 S. Bhowmick, S. Rother, H. Zimmermann, P. S. Lee, S. Moeller, M. Schnabelrauch, V. Koul, R. Jordan, V. Hintze and D. Scharnweber, Mater. Sci. Eng., C, 2017, 79, 15-22.

51 J. Iida, J. Dorchak, R. Clancy, J. Slavik, R. Ellsworth, Y. Katagiri, E. N. Pugacheva, T. H. Van Kuppevelt, R. J. Mural, M. L. Cutler and C. D. Shriver, Exp. Cell Res., 2015, 330(2), 358-370. 
52 S. C. H. A. Van der Steen, A. A. G. van Tilborg, M. J. E. Vallen, J. Bulten, T. H. Van Kuppevelt and L. F. A. G. Massuger, Gynecol. Oncol., 2016, 140(3), 527-536.

53 W. Suchaoin, S. Bonengel, J. A. Griebinger, I. Pereira de Sousa, S. Hussain, C. W. Huck and A. Bernkop-Schnurch, Eur. J. Pharm. Biopharm., 2016, 101, 25-32.

54 K. Yoshirou, S. Nobuo, K. Koji, K. Makoto and K. Yoshimitsu, FEBS Lett., 2013, 587, 3943-3948.

55 C. B. Leticia, F. M. Alessandro, C. B. Ismael, P. Francielle, V. N. Celso, F. R. Adley and C. M. Edvani, Int. J. Pharm., 2014, 477, 197-207.

56 P. Melgar-Lesmes, F. Garcia-Polite, P. Del-Rey-Puech, E. Rosas, L. D. Juliana, E. Montell, V. Josep, R. E. Elazer and B. Mercedes, Atherosclerosis, 2016, 245, 82-87.

57 H. Yimin, C. Jingdi, F. Tiantang, Z. Yujue, Z. Yao, S. Xuetao and Z. Qiqing, Colloids Surf., B, 2017, 157, 93-100.

58 L. Shaojun, D. Yan, X. Zhen, H. Haobo, Z. Aijun, L. Li, Y. Yan and L. Quanshun, Colloids Surf., B, 2015, 136, 577-584.
59 N. Yilu, T. Zhurong, C. Wanxu, L. Hai, F. Yujiang, G. Likun and Z. Xingdong, Int. J. Biol. Macromol., 2015, 74, 367-375.

60 W. Mingyi, W. Dandan, G. Na, X. Chuang, Y. Lian, X. Li, L. Wu, P. Wenlie, J. Jianmin and Z. Jinhua, Euro J Med Chem, 2015, 92, 257-269.

61 L. M. Loeb, M. G. Naffah-Mazzacoratti, M. A. Porcionatto, J. R. M. Martins, M. Kouyoumdjian, L. M. Weckx and H. B. Nader, Int. Immunopharmacol., 2008, 8, 1056-1058.

62 N. Satomi, K. Hiroki and K. Hiroshi, J. Biol. Chem., 2017, 293, 444-465.

63 K. C. Butterfield, A. W. Conovaloff and A. Panitch, Biomatter, 2011, 1, 174-181.

64 F. J. V. Pahi, J. V. Milano and M. P. Lopez, WO2005014012A1, World Int Property Org, 2005.

65 P. Suleyman, Euro Pat., 15174427.3 EP3111941, 2017.

66 A. Aspberg, D. HeinegArd, A. Johnson and A. Kist, US Pat., US7956047B2, 2005. 